\title{
Pompéi - Nécropole romaine de Porta Nocera : la campagne 2020
}

\section{William Van Andringa}

\section{OpenEdition}

\section{Journals}

Édition électronique

URL : https://journals.openedition.org/baefe/3218

DOI : $10.4000 /$ baefe.3218

ISSN : 2732-687X

Éditeur

ResEFE

Référence électronique

William Van Andringa, « Pompéi - Nécropole romaine de Porta Nocera : la campagne 2020 » [notice archéologique], Bulletin archéologique des Écoles françaises à l'étranger [En ligne], Italie, mis en ligne le 27 janvier 2022, consulté le 29 janvier 2022. URL : http://journals.openedition.org/baefe/3218 ; DOI : https://doi.org/10.4000/baefe.3218

Ce document a été généré automatiquement le 29 janvier 2022.

\section{c)}

Le Bulletin archéologique des Écoles françaises à l'étranger est mise à disposition selon les termes de la Licence Creative Commons Attribution - Pas d'Utilisation Commerciale - Pas de Modification 4.0 International. 


\title{
Pompéi - Nécropole romaine de Porta Nocera : la campagne 2020
}

\author{
William Van Andringa
}

\section{NOTE DE L'AUTEUR}

Autorité nationale présente : Parco archeologico di Pompei

Composition de l'équipe de terrain : Philippe Brunner (UMR 5140 ASM Montpellier); Anselme Cormier (CHUS, Université catholique de l'Ouest, Angers); Sandra Dal Col (Eveha) ; Franck Decanter (UMR 8546 CNRS ENS-Paris AOrOc) ; Henri Duday (UMR 5199 du CNRS PACEA, université de Bordeaux) ; François Fouriaux (EFR, UMR 8546 CNRS ENS-Paris AOrOc) ; Flore Giraud ; Chloé Lacourarie ; Tuija Lind ; Paloma Lorente Sebastián ; Adrien Malignas (Mosaïques Archéologie, UMR 8546 CNRS ENS-Paris AOrOc) ; Véronique Matterne (CNRS / MNHN) ; Sophie Pillault ; Émilie Portat (Direction de l'Archéologie, Chartres Métropole, UMR 7041 ArScAn) ; Audrey Roger (université de Toulouse Jean-Jaurès) ; Verdiana Sorrento (Eveha) ; William Van Andringa (EPHE, UMR 8546 CNRS ENS-Paris AOrOc et IUF)

Établissement éditeur : EFR

Établissements porteurs de l'opération et partenariats : École française de Rome ; École Pratique des Hautes Études (UMR 8546 CNRS ENS-Paris AOrOc, Université PSL) Parco Archeologico di Pompei ; ARPAMED ; Institut Universitaire de France ; UMR 5199 du CNRS PACEA.

Remerciements : Nous tenons à remercier le professeur directeur Massimo Osanna et la dott.ssa Marialaura Iadanza du Parc archéologique ainsi que Brigitte Marin, directrice de l'École Française de Rome et Nicolas Laubry, directeur des études, pour leur soutien accordé à ce programme de recherches. Notre gratitude va également à Evelyne Bukowiecki et à François Fouriaux du laboratoire d'archéologie de l'École française de Rome pour leur aide précieuse dans le montage, désormais complexe, de la demande de concession. L'organisation de la campagne a bénéficié également de l'implication précieuse d'Ilaria Parisi, assistante scientifique (EFR), d'Isabelle Mariage, secrétaire générale (AOrOc) et d'Henri de Mégille (Arpamed). Ce chantier 
programmé a bénéficié des subventions accordées par l'École française de Rome, l'Institut Universitaire de France, les laboratoires AOrOc et PACEA ainsi que par le fonds de dotation Arpamed sans qui la réalisation de la recherche programmée n'aurait pas pu voir le jour. Nous remercions chaleureusement ces institutions.

Concessione DG-ABAP 768 del 10/06/2020 stipulata con il Ministero per i Beni e le Attività culturali.

Données scientifiques produites :

Rapport d'opération (W. Van Andringa et H. Duday (dir.), Pompéi - Fouille de la nécropole romaine de Porta Nocera : campagne 2020, Rapport final d'opération, Pompéi, 2020)

\section{Chroniques de l'EFR :}

Pompéi - Fouille de la nécropole romaine de Porta Nocera 2020 (2019)

La nécropole romaine de Porta Nocera à Pompéi 2019 (2018)

La nécropole romaine de Porta Nocera à Pompéi 2018 (2017)

La nécropole romaine de Porta Nocera à Pompéi 2017 (2016)

La nécropole romaine de Porta Nocera à Pompéi 2016 (2015)

La nécropole romaine de Porta Nocera à Pompéi 2015 (2014)

1 La campagne 2020 s'est déroulée du 31 août au 11 octobre, soit sur six semaines pleines. La première partie de l'opération, organisée du 31 août au 27 septembre, a concerné la fouille de plusieurs secteurs funéraires. La priorité de cette année était l'achèvement, dans la zone B, de la fouille des enclos 3E (Sandra Dal Col, Paloma Lorente et Sophie Pillault) et 3D (Emilie Portat et Tuija Lind) ainsi que la continuation de l'étude exploratoire de l'aire funéraire 1J (François Fouriaux, Chloé Lacourarie, Audrey Roger, Verdiana Sorrento et William Van Andringa). La nécessité de déployer des enregistrements rigoureux et chronophages, essentiels pour la restitution des séquences funéraires, n'a pas permis d'achever la fouille des aires 3E et 3D. Néanmoins, la présence sur le terrain a été prolongée presque jusqu'au terme de la campagne de manière à quasiment achever le travail, si bien qu'il est désormais envisageable de finir la fouille de ces deux enclos lors de la prochaine mission: deux ou trois semaines seulement, prévues en septembre 2021, devraient être suffisantes pour compléter les enregistrements de 3E et 3D. Pourront alors commencer le traitement des données et l'étude de l'aire de crémation et des 17 sépultures de $3 \mathrm{E}$, également la comparaison des modus operandi déployés pour les 13 sépultures installées dans l'aire 3D. Il s'agira en particulier de voir le détail des séquences de mise au tombeau pour chacune des tombes, pour saisir la variabilité des pratiques funéraires au sein d'un même groupe familial.

\section{L'aire funéraire 3E (fig. 1)}

2 En 2020, trois personnes ont été de nouveau mobilisées pour continuer la fouille de l'enclos 3E, situé sur la terrasse bordant au sud la route de Nocera. En surface, l'aire de crémation se caractérise par un plan circulaire plus sombre que le remblai dans lequel les sépultures de l'enclos s'installent. Ses dimensions sont de 1,96 m maximum d'est en ouest et 2,17 m maximum du nord au sud, soit environ $3,9 \mathrm{~m}^{2}$, dans un enclos occupant une surface totale de $11,95 \mathrm{~m}^{2}$. La présence d'éléments osseux brulés, de charbons et 
d'un très grand nombre d'ossements ouvragés a très vite aiguillé les interprétations vers la caractérisation d'une aire de crémation centrale fonctionnant avec les sépultures de l'enclos.

Le travail a consisté exclusivement à la reprise de la partie centrale de l'aire occupée par l'aire de crémation AC16 qui s'est révélée être dans sa dernière phase une sépulture primaire à crémation, la SP 3E18. En 2019, l'aire de crémation AC16 a été fouillée jusqu'aux niveaux appartenant à la sépulture primaire 3E18 dont l'amas osseux et les résidus de bûchers ont été intégralement dégagés et fouillés. En 2020, la fouille a donc concerné les derniers niveaux (5345 et 5346) ainsi que les parois rubéfiées du bûcher. Il s'agissait également de savoir si les restes de structures antérieures avaient été préservés en fouillant les banquettes latérales de la structure (5328 et 5333).

Fig. 1. Enclos 3E : la sépulture primaire à crémation en cours de fouille.

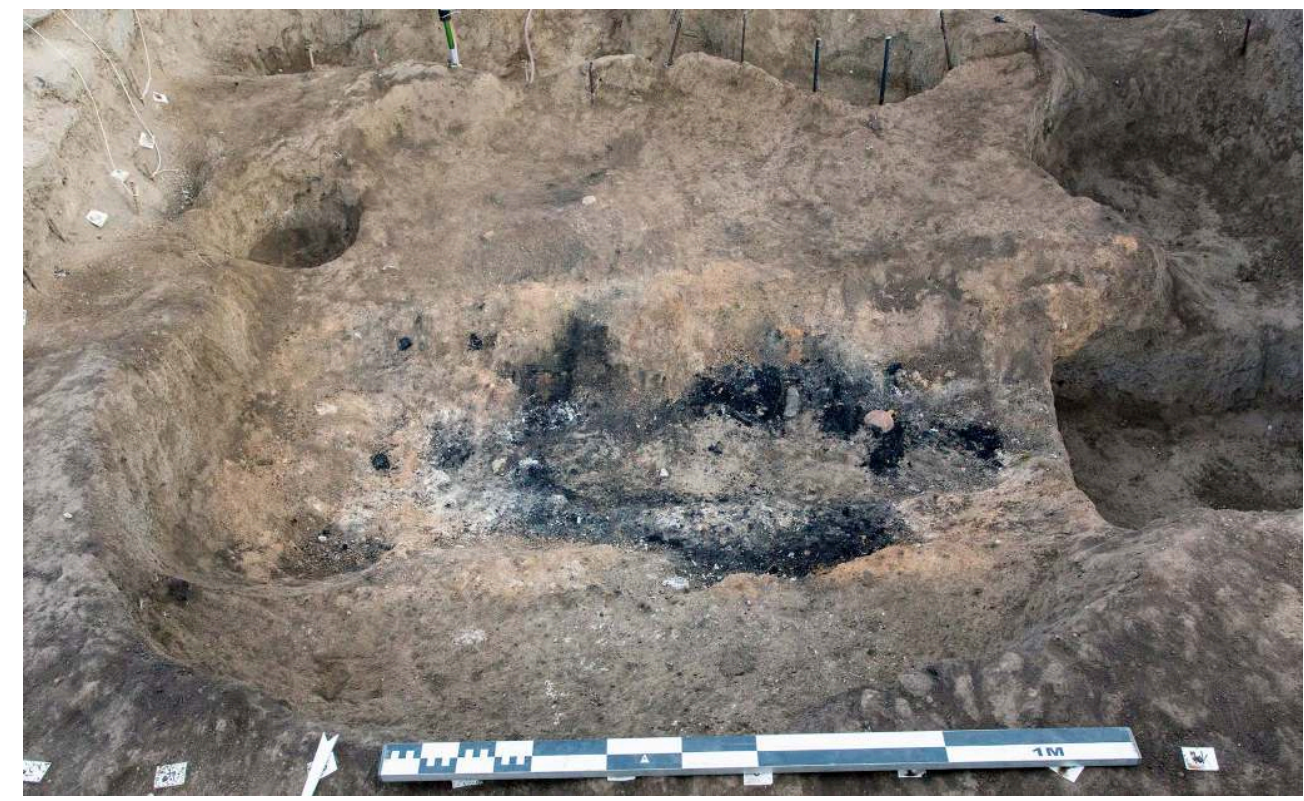

Sur la partie droite du foyer, on voit la lampe à huile retournée et brisée, un geste qui marque la fin de la cérémonie des funéraille.

F. Giraud.

\section{L'aire funéraire 3D (fig. 2)}

Deux personnes ont continué la fouille de l'aire 3D, mitoyenne à l'ouest de l'enclos 3E. Six sépultures et un dépôt indéterminé ont été enregistrées (sur un total de 13 sépultures et 15 structures) : SP9, SP4, SP5, SP6, SP12, SP13 et ST14. La SP9 est une sépulture à bâtière munie d'un tube à libations et surmontée d'une stèle en marbre clouée dans le mur de l'enclos. Les résidus du bûcher ont été disposés au fond de la structure avant qu'un sac contenant les restes osseux brûlés ne soient déposés au centre de la tombe. La particularité de cette sépulture est qu'une quarantaine de balsamaires en verre ont été glissés après usage dans le tube à libations. La mince couche d'infiltration relevée entre l'amas osseux et les premiers flacons indique que ce dépôt est intervenu après la fermeture de la tombe, lors des visites faites au défunt. Le colmatage du tube par des pierres ponces indique que celles-ci ont été interrompues 
par l'éruption de 79. Les autres sépultures fouillées s'organisent de part et d'autre du lot funéraire dominé par une tombe dont le marquage monumental (stèle et plaque de scellement) indique qu'elle est plus importante que les autres. Cette sépulture qui est peut-être la tombe fondatrice sera étudiée en 2021 , lors de la dernière campagne dévolue à cette aire funéraire.

Fig. 2. Enclos 3D : treize sépultures dans six mètres carrés.

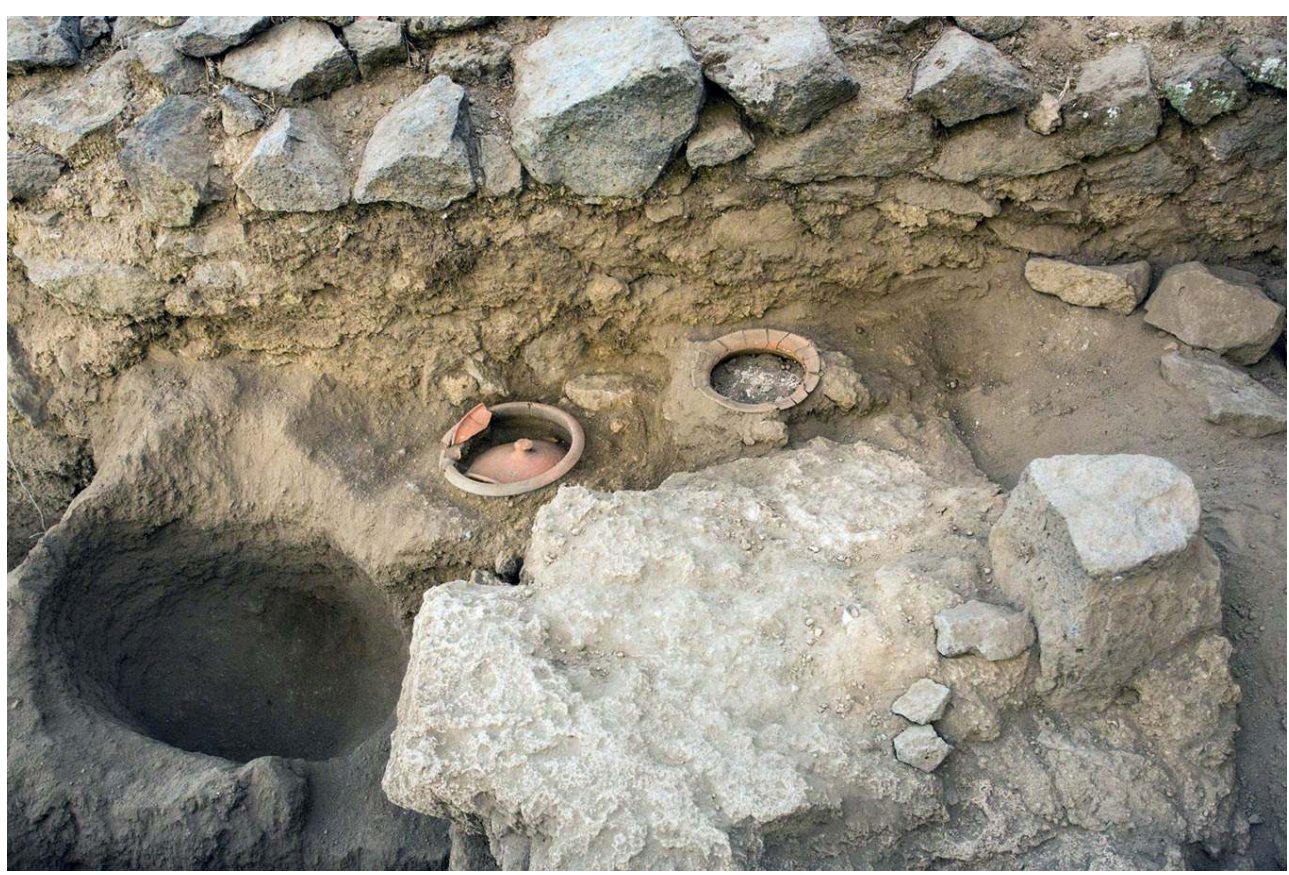

Les tombes sont alignées de part et d'autre de la sépulture fondatrice, au premier plan, constituée d'un caisson fait de grandes dalles de calcaire.

F. Giraud.

\section{L'aire funéraire $1 \mathrm{~J}$ (fig. 3)}

En 2020, la fouille s'est poursuivie dans l'aire funéraire 1J située au nord de la route de Nocera, entre l'enclos 1F des Veranii et le monument G. Comme les autres années, l'espace en question, occupé par une épaisse couche d'occupation en place et trois sépultures marquées par des stèles en lave a été étudié en mode " laboratoire ", avec un protocole poussé de collecte et de géolocalisation des artéfacts et écofacts, permettant de retracer l'activité funéraire (et non funéraire) déployée. La géolocalisation du mobilier ou des témoins a été complété cette année par un enregistrement de chaque fait visible après chaque passe : ce découpage en unités stratigraphiques est destiné à mieux comprendre les phénomènes sédimentaires et anthropiques de sectorisation. Deux à trois passes de fouille ont ainsi été effectuées sur une zone désormais bien circonscrite au nord par la présence d'une tranchée moderne et de porter le nombre d'objets ou de témoins isolés à plus de 3803 (sur le protocole, voir Annexe 1). 
Fig. 3. Aire funéraire $1 \mathrm{~J}$.

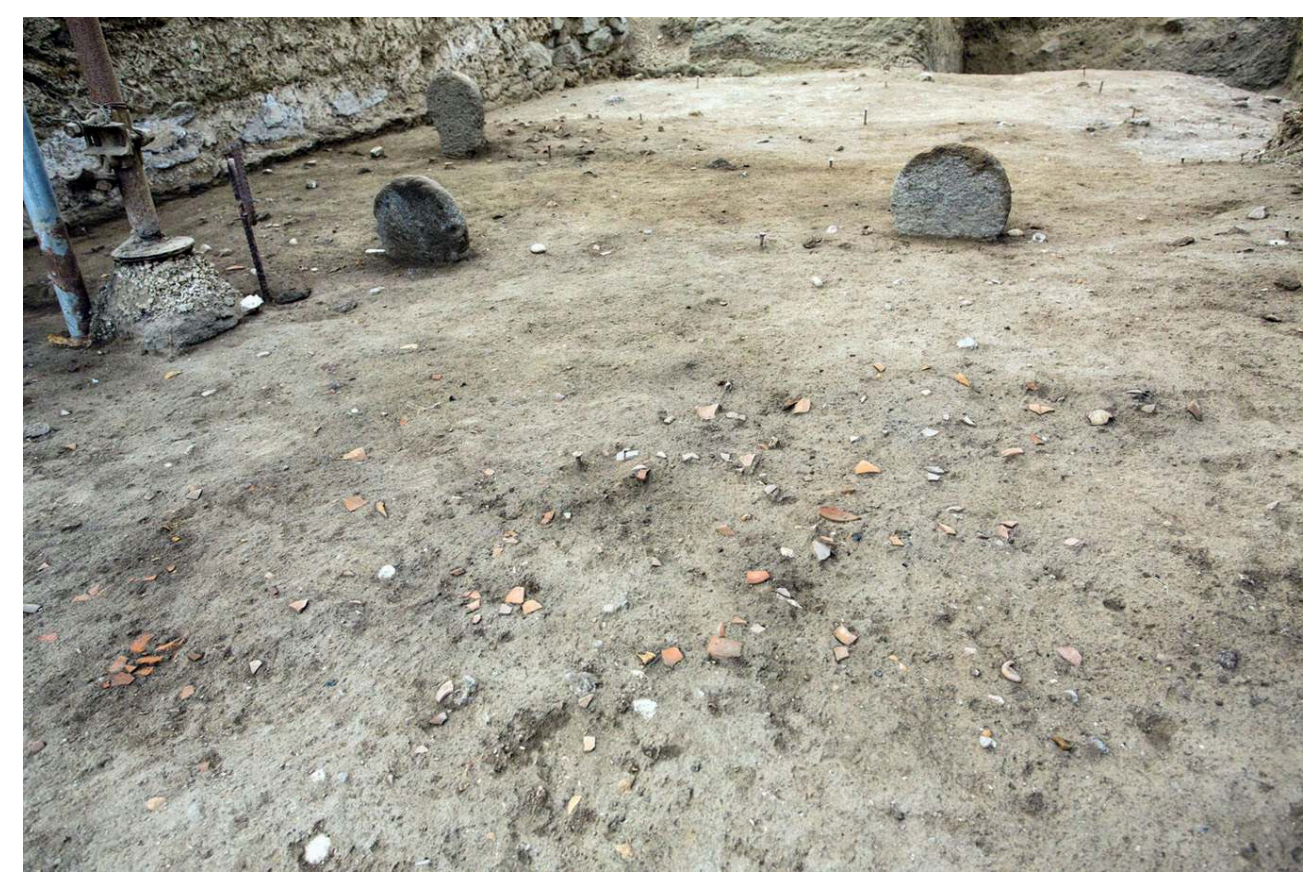

Des dizaines de fragments de gobelets à paroi fine jonchent le sol, témoignant de rites célébrés auprès des sépultures disposées au second plan.

F. Giraud.

\section{Fouille d'évaluation des enclos 9ES et de M. Tullius}

6 En complément de ces travaux menés depuis 2015 dans le secteur dit « Fondo Pacifico », une fouille d'évaluation a été conduite dans l'enclos 9ES appartenant à C. Munatius Faustus. L'objectif était de confirmer le bon état des structures funéraires enfouies, sachant que la Surintendance a fait enlever les stèles funéraires de l'enclos lors d'une opération mal datée des années 80 ou 90 . Deux jours ont suffi : le sondage implanté sur un peu plus du quart de la surface de l'enclos a montré que les tombes et la couche d'occupation étaient en place malgré l'arrachage des stèles ; la fouille s'est donc arrêtée sur le sol le plus récent ; elle sera reprise en 2021 dans le cadre d'une étude spécifique sur les affranchis et la mort. Un autre sondage d'évaluation a intéressé l'aire funéraire attenante au monument funéraire de M. Tullius à la porte de Stabies. Là encore, le décapage sur quelques centimètres d'un carré de $2 \times 2$ mètres a suffi à mettre au jour un remblai antique en place sous la terre végétale et une mince couche détritique moderne. Les diagnostics étant positifs dans les deux cas, l'étude de ces aires funéraires pourra être menée en 2021.

\section{Session d'étude des os humains et du mobilier}

7 Parallèlement à la fouille, A. Malignas a travaillé deux semaines sur les lots céramiques de l'enclos $1 \mathrm{~F}$, ce qui lui a permis de mettre en évidence des emplois distincts du parfum lors de la séquence de crémation et de collecte des os des défunts du lot funéraire. Il s'agit là d'un apport majeur dans la compréhension de cette «danse à trois » des acteurs funéraires constituée par l'officiant, l'assistance et le défunt. 
8 La fouille a été suivie d'une campagne d'étude des os humains et du matériel. L'effort a surtout porté sur le matériel archéologique de l'enclos $1 \mathrm{~F}$ : l'étude des ossements humains (Henri Duday avec la collaboration de Philippe Bruner), des ossements animaux (Franck Decanter), de la céramique (Adrien Malignas), des os ouvragés (Anselme Cormier) et des restes carpologiques (Véronique Matterne) étant désormais achevée pour cette aire funéraire, il est possible de programmer le rendu d'un manuscrit pour la fin de l'année 2021.

\section{INDEX}

sujets https://ark.frantiq.fr/ark:/26678/pcrt69L0JofMDy, https://ark.frantiq.fr/ark:/26678/ pcrtsIm3RuNMGu, https://ark.frantiq.fr/ark:/26678/pcrt795b632nWw

Année de l'opération : 2020

Thèmes : EFR

lieux https://ark.frantiq.fr/ark:/26678/pcrtPz1h9HJaSX, https://ark.frantiq.fr/ark:/26678/ pcrt7ya9W00Xhb

\section{AUTEURS}

\section{WILLIAM VAN ANDRINGA}

École Pratique des Hautes Études, Paris ; UMR 8546 CNRS ENS-Paris AOrOc et IUF

\section{DIRECTEURFOUILLES_DESCRIPTION}

\section{WILLIAM VAN ANDRINGA}

École Pratique des Hautes Études, Paris ; UMR 8546 CNRS ENS-Paris AOrOc et IUF 